\title{
A note on information revelation in procurement auctions*
}

\author{
Nicola Doni \\ (nicola.doni@unifi.it) \\ Dipartimento di Scienze Economiche \\ Università degli Studi di Firenze, Via delle Pandette 9, I-50127 Firenze, Italy \\ AND \\ Domenico Menicucci** \\ (domenico.menicucci@dmd.unifi.it) \\ Dipartimento di Matematica per le Decisioni \\ Università degli Studi di Firenze, Via C. Lombroso 6/17, I-50134 Firenze, Italy
}

February 22, 2010

\begin{abstract}
This paper is about a procurement auction setting, introduced in Gal-Or, Gal-Or and Dukes (2007), in which suppliers offer differentiated products and the buyer needs to decide whether to reveal or not to the suppliers the own preferences for the various products. We provide some technical remarks and complements to the analysis of Gal-Or, Gal-Or and Dukes (2007), and an extension to the case of risk averse suppliers.
\end{abstract}

JEL Classification: D44, D82.

Key words: Information Revelation, Logconcavity, Risk Aversion.

${ }^{*}$ We thank Andrey Sarychev for useful comments and suggestions. The usual disclaimer applies.

**Please send mail to Domenico Menicucci, Dipartimento di Matematica per le Decisioni, via C. Lombroso 6/17, I-50134 Firenze (FI), Italy; e-mail address: domenico.menicucci@dmd.unifi.it; phone: +39-055-4796773; fax: +39-055-4796800. 


\section{Introduction}

This paper is about a buyer's incentives to reveal the own preferences to the suppliers in a procurement auction. We rely on a setting which is studied in Gal-Or, Gal-Or and Dukes (2007) (GGD henceforth) and provide some technical remarks to their analysis, complements and extensions which are described in the second half of this introduction.

GGD consider a setting in which a male buyer denoted with B needs to buy a certain object (for instance, an industrial firm needs to procure an input) and faces $n \geq 2$ female suppliers which can provide the object. The products offered by the suppliers are differentiated and for each supplier $i$ there is a parameter $x_{i} \in[0,1]$ which represents the degree of fitness of $i$ 's product with B's needs; precisely, if B buys that object and pays $p$ then his payoff is $x_{i}-p$. $\mathrm{B}$ is risk neutral and uses a first score auction in which suppliers simultaneously submit bids $p_{1} \geq 0, \ldots, p_{n} \geq 0$, and then B buys product $i$ such that $x_{i}-p_{i}>x_{j}-p_{j}$ for any $j \neq i$; ties can be broken arbitrarily. ${ }^{1}$ Before running the auction, B observes the values $x_{1}, \ldots, x_{n}$ but suppliers do not: each supplier views $x_{1}, \ldots, x_{n}$ as the realizations of $n$ i.i.d. random variables, each with the same absolutely continuous c.d.f. $F$ and strictly positive density $f=F^{\prime}$ on $(0,1)$.

Before observing $x_{1}, \ldots, x_{n}$, B has the same beliefs as the suppliers about his values for the objects, and GGD inquire whether at this stage B should commit to a policy of no information revelation (concealment policy, henceforth denoted by $\mathrm{C}$ ), or instead to a policy in which he will reveal to each supplier $i$ the value of $x_{i}$ (private revelation policy, henceforth denoted by $\mathrm{PR}){ }^{2}$ Clearly, under PR supplier $i$ may charge a premium price trying to extract B's surplus, especially if $x_{i}$ is large, but $\mathrm{PR}$ also intensifies price competition since a low fitness supplier needs to reduce her bid to be competitive, and this may force also high fitness suppliers to compete aggressively. Hence, whether B prefers PR or C is not obvious; we use $\sim$ and $\succ$ to represent B's preferences between $\mathrm{C}$ and $\mathrm{PR}$.

A major claim in GGD is that if the density $f$ is (weakly) increasing, then $\mathrm{PR} \succ \mathrm{C}$. However, in Section 2 we give an example in which $f$ is increasing but $\mathrm{C} \succ \mathrm{PR}$. This apparent inconsistency occurs because the proof of GGD's result relies on the assumption (unstated in the main text of the paper) of logconcavity for the c.d.f. $F$ : our example violates logconcavity of $F$ and thus the simple property of increasing $f$ does not imply $\mathrm{PR} \succ \mathrm{C} .{ }^{3}$

In order to analyze regime C, GGD focus on pure strategy symmetric Nash Equilibrium, but they neglect that in some cases no such equilibrium exists. We point out that Caplin and

\footnotetext{
${ }^{1}$ In fact, GGD also study a model in which B approaches suppliers sequentially and needs to derive the optimal stopping rule. We do not consider this setting in our paper.

${ }^{2}$ While this commitment assumption may appear strong, GGD notice that B may choose between providing a detailed specification of the attributes of the object he wishes to procure (which allows each supplier $i$ to infer $x_{i}$ ), and providing instead vague or minimal details (which leaves each supplier in the dark).

${ }^{3}$ Our example is relevant also because GGD fail to find a distribution such that $\mathrm{C} \succ \mathrm{PR}$.
} 
Nalebuff (1991) provide sufficient conditions for existence in a more general model. Furthermore, we notice that when $n=2$ the property that $f$ is increasing has the same effect on the comparison between $\mathrm{C}$ and $\mathrm{PR}$ as the property that $f$ is decreasing; in a sense, this is at odds with the focus of GGD on increasing densities.

Finally, in Section 3 we consider the case of risk averse suppliers with constant relative risk aversion, and show that $\mathrm{C} \succ \mathrm{PR}$ under strong risk aversion, regardless of the distribution. The proofs are given in the appendix.

\section{A few remarks and complements to the analysis of GGD}

GGD assume that suppliers are risk neutral and, for regime $\mathrm{C}$, they consider symmetric pure strategy Nash equilibria (NE henceforth), that is NE in which all suppliers make the same bid $\hat{p}>0$ [if $\hat{p}=0$, then each supplier earns zero but can make a positive profit with a bid in $(0,1)$. In order to derive $\hat{p}$, GGD study supplier $i$ 's optimal choice of her own bid $p_{i}$, given that each other supplier plays $\hat{p}$. Since $i$ wins if and only if $x_{i}-p_{i}>x_{j}-\hat{p}$ for any $j \neq i$ (neglecting zero probability events), and she does not observe $\left(x_{1}, \ldots, x_{n}\right), i$ evaluates her own winning probability as $\int_{0}^{1} F^{n-1}\left(x_{i}+\hat{p}-p_{i}\right) f\left(x_{i}\right) d x_{i}$ and $i$ 's payoff as a function of $p_{i}$ is $\pi_{i}\left(p_{i}\right) \equiv p_{i} \int_{0}^{1} F^{n-1}\left(x_{i}+\hat{p}-p_{i}\right) f\left(x_{i}\right) d x_{i}$ (each supplier bears no production cost). GGD impose that the FOC for maximizing $\pi_{i}$ with respect to $p_{i}$ is satisfied at $p_{i}=\hat{p}$ and find

$$
\hat{p} \equiv \frac{1}{n \int_{0}^{1}(n-1) F^{n-2}(x) f^{2}(x) d x}
$$

This implies that the unique candidate for a symmetric pure strategy NE is the profile in which each supplier bids $\hat{p}$ in (1), denoted by $\hat{\mathbf{p}}$ in the following. Given $\hat{\mathbf{p}}$, the highest fitness supplier wins. The problem with this methodology is that merely satisfying a FOC does not guarantee that a maximum point for $\pi_{i}$ is obtained. Indeed, in some cases $\hat{\mathbf{p}}$ is not a NE, and then no symmetric pure strategy NE exists.

Example 1 Suppose that $n=2$ and $f(x)=\frac{2}{3} x^{-1 / 3}, F(x)=x^{2 / 3}$; then $\hat{p}=\frac{3}{8}$. However, given $p_{2}=\frac{3}{8}$, the payoff of supplier 1 when $p_{1} \geq \frac{3}{8}$ is $\pi_{1}\left(p_{1}\right)=p_{1} \int_{p_{1}-\frac{3}{8}}^{1}\left(x_{1}+\frac{3}{8}-p_{1}\right)^{2 / 3} \cdot \frac{2}{3} x_{1}^{-1 / 3} d x_{1}$ and numeric integration gives $\pi_{1}\left(\frac{45}{100}\right) \simeq \frac{19}{100}>\pi_{1}\left(\frac{3}{8}\right)=\frac{3}{16}$. Thus $\hat{\mathbf{p}}=\left(\frac{3}{8}, \frac{3}{8}\right)$ is not a NE. ${ }^{4}$

Example 1 suggests some caution with GGD's analysis for regime $\mathrm{C}$, but under suitable conditions it is possible to prove that $\hat{\mathbf{p}}$ is a NE. Indeed, Caplin and Nalebuff (1991) (CN henceforth) provide an existence theorem for models of price competition with differentiated products which include GGD's C setting. In particular, Theorem 2 in CN implies that $\hat{\mathbf{p}}$ is a

\footnotetext{
${ }^{4}$ Perloff and Salop (1985) consider a model of product differentiation which is formally equivalent to GGD's $\mathrm{C}$ setting. They notice that a symmetric pure strategy NE may not exist, but in their counterexample $F$ has a mass point and thus is not absolutely continuous.
} 
$\mathrm{NE}$ as long as $f$ is logconcave (in fact, a somewhat weaker condition suffices). ${ }^{5}$

In the regime of $\mathrm{PR}, \mathrm{B}$ privately reveals to supplier $i$ (for $i=1, \ldots, n$ ) the signal $x_{i}$. GGD consider symmetric Bayes-Nash equilibria (BNE henceforth), that is BNE in which each supplier bids according to the same bidding function $P:[0,1] \rightarrow \mathbb{R}$, which depends on the signal the supplier learns from B. GGD identify a symmetric BNE in which

$$
P(x)=\int_{0}^{x} \frac{F^{n-1}(z)}{F^{n-1}(x)} d z
$$

Supplier $i$ wins if and only if $x_{i}-P\left(x_{i}\right)>x_{j}-P\left(x_{j}\right)$ for any $j \neq i$, and since $x_{i}-P\left(x_{i}\right)>$ $x_{j}-P\left(x_{j}\right)$ if and only if $x_{i}>x_{j}$, it follows that the highest fitness supplier wins under PR. ${ }^{6}$

Given that B selects the supplier with the highest fitness in both regimes C and PR, B prefers the regime in which his expected payment is lower. In the case of $\mathrm{C}, \mathrm{B}$ pays simply $\hat{p}$. In PR, B's expected payment is $\int_{0}^{1} P(x) d F^{n}(x)$, the expected bid of the highest fitness supplier. Simple manipulations reveal that $\int_{0}^{1} P(x) d F^{n}(x) \leq \hat{p}$, that is $\mathrm{PR} \succeq \mathrm{C}$, if and only if

$$
n \int_{0}^{1} F^{n-1}(x)[1-F(x)] d x \int_{0}^{1}(n-1) F^{n-2}(x) f^{2}(x) d x \leq \frac{1}{n}
$$

Proposition 2 in GGD claims that when $f$ is increasing, the inequality $P(x) \leq \hat{p}$ holds for any $x \in[0,1]$. An obvious corollary is then as follows [Proposition 3(ii) in GGD]: If $f$ is increasing, then (3) holds strictly. However, the proof of Proposition 2 uses both the assumption of $f$ increasing and of logconcavity for $F$; thus, such a proposition should be stated as follows: If $F$ is logconcave and $f$ is increasing, then $P(x) \leq \hat{p}$ for any $x \in[0,1]$; the corollary, Proposition 3(ii), should be stated as follows:

$$
\text { If } F \text { is logconcave and } f \text { is increasing, then } \mathrm{PR} \succ \mathrm{C}
$$

Our example 2 below is such that $f$ is increasing, $F$ is not logconcave and (3) is violated, which means that $\mathrm{C} \succ \mathrm{PR}$. Therefore it cannot be proved that $\mathrm{PR} \succ \mathrm{C}$ under the sole assumption that $f$ is increasing; a fortiori, the same assumption alone does not imply that $P(x) \leq \hat{p}$ for any $x \in[0,1]$. Example 2 is of interest also because GGD cannot find an example such that $\mathrm{C} \succ \mathrm{PR}$.

Example 2 Suppose that $n=2$ and

$$
f(x)=\left\{\begin{array}{ll}
\frac{1}{2} & \text { for } x \in\left[0, \frac{4}{5}\right] \\
3 & \text { for } x \in\left(\frac{4}{5}, 1\right]
\end{array}, \quad F(x)=\left\{\begin{array}{cl}
\frac{1}{2} x & \text { for } x \in\left[0, \frac{4}{5}\right] \\
3 x-2 & \text { for } x \in\left(\frac{4}{5}, 1\right]
\end{array}\right.\right.
$$

\footnotetext{
${ }^{5}$ The density $f$ is said to be $\log$ concave if and only if $\log f$ is a concave function. In fact, in CN the set of feasible prices for each supplier is $[0, y]$, where $y>0$ is a fixed parameter, but for GGD's C setting existence can be proved, under $f$ logconcave, even though there is no maximal price; details are available in the appendix. See Section 6.3 in Anderson, de Palma and Thisse (1992) for a very readable presentation of CN's result.

${ }^{6}$ The analysis of GGD can be simplified by noticing that this setting is closely related to the well known environment in which an object is sold through a first price auction with $n$ bidders: we can prove that there is a one-to-one correspondence between the BNE in the two settings. Then (2) is obtained straightforwardly.
} 
We find that $\hat{p}=\frac{1}{4}$, but $f$ is not logconcave [since $\log f$ is not continuous in $\left.(0,1)\right]$ and thus we cannot apply the results of $\mathrm{CN}$ to infer that $\left(p_{1}, p_{2}\right)=\left(\frac{1}{4}, \frac{1}{4}\right)$ is a NE under C; however, we can prove this result directly. ${ }^{7}$ Given this fact, we compare $\mathrm{C}$ and PR by verifying that (3) reduces to $2 \cdot \frac{23}{150} \cdot 2 \leq \frac{1}{2}$, which is violated.

In order to understand how much the result as stated in (4) is more restrictive with respect to the formulation in GGD, it is useful to notice that $F$ logconcave is equivalent to $\frac{f}{F}$ decreasing, which fails to hold if $f$ increases too quickly. Thus the assumption in (4) is that $f$ is increasing but nowhere too quickly. ${ }^{8}$

When they provide an intuition on why an increasing $f$ favors PR against C, GGD argue that in such a case a high fitness supplier is likely to face competitors with high fitness, and this induces her to bid low under PR. The opposite argument applies if $f$ is decreasing, since then a high fitness supplier likely faces low fitness competitors and thus she may want to bid less aggressively. This intuition is incomplete, as it neglects the effect of $f$ on $\hat{p}$, and furthermore the next proposition casts some doubts on it when $n=2$. To this purpose, we define

$$
g(x) \equiv f(1-x), \quad G(x) \equiv \int_{0}^{x} g(z) d z \quad \text { for any } x \in[0,1]
$$

It is simple to see that $g$ is a density function on $[0,1]$ and $G$ is its c.d.f.; moreover, the graph of $g$ is the mirror image of the graph of $f$ with respect to the vertical line $x=\frac{1}{2}$.

Proposition 1 Given an arbitrary density $f$ and c.d.f. $F$, if $g$ and $G$ are defined as in (5) then $2 \int_{0}^{1} F(x)[1-F(x)] d x \int_{0}^{1} f^{2}(x) d x=2 \int_{0}^{1} G(x)[1-G(x)] d x \int_{0}^{1} g^{2}(x) d x$. Thus, when $n=2$ the left hand side of (3) is unchanged if $f$ is replaced by its mirror image with respect to $x=\frac{1}{2}$.

As a consequence, if $f$ is increasing then $g$ is decreasing and it satisfies or violates (3) just like $f$ does. Therefore, when $n=2$, satisfying (3) with an increasing density is just "as simple as" satisfying (3) with a decreasing density.

\section{The effect of risk aversion}

In this section we consider the case in which suppliers are risk averse with a utility function $u$ which is strictly increasing, strictly concave and such that $u(0)=0$. In particular we analyze how a specific form of risk aversion affects B's preferences between $\mathrm{C}$ and PR.

\footnotetext{
${ }^{7}$ The proof is available in the appendix. Indeed, finding a distribution which violates (3) is relatively straightforward, but it is quite less simple to find a distribution such that in addition $\hat{\mathbf{p}}$ is a NE under C.

${ }^{8}$ It is worthwhile to notice that when $f$ is logconcave we have that (i) $\hat{\mathbf{p}}$ is a NE in regime C; (ii) $F$ is logconcave in view of Lemma 3 in An (1998). Thus, when $f$ is logconcave it is correct to claim that $f$ increasing implies $\mathrm{PR} \succ \mathrm{C}$. But GGD make no assumption about the logconcavity of $f$.
} 
Proposition 2 (i) Suppose that suppliers are risk averse. If a symmetric pure strategy NE exists under concealment, then it is unique and such that each supplier bids $\hat{p}_{r}$ which satisfies

$$
\frac{u\left(\hat{p}_{r}\right)}{u^{\prime}\left(\hat{p}_{r}\right)}=\hat{p}
$$

and $\hat{p}_{r}<\hat{p}$. If $f$ is logconcave, then the profile of bids in which each supplier bids $\hat{p}_{r}$ is a NE.

(ii) Under private revelation, in any symmetric pure strategy BNE each supplier with type $x$ bids $P_{r}(x)$, in which the function $P_{r}$ satisfies

$$
P_{r}^{\prime}(x)=1-\frac{u\left[P_{r}(x)\right]}{u^{\prime}\left[P_{r}(x)\right]}(n-1) \frac{f(x)}{F(x)}
$$

and $P_{r}(x)<P(x)$ for any $x \in(0,1]$.

Proposition 2 reveals that risk averse suppliers are more aggressive than risk neutral suppliers, both in regime $\mathrm{C}$ and in regime $\mathrm{PR} .{ }^{9}$ The intuition for this result is that a risk averse supplier bids more aggressively than a risk neutral supplier because she tries to win with a higher probability, albeit with a smaller profit. ${ }^{10}$

We now consider the particular case in which $u(p)=p^{a}$ for some $a \in(0,1)$, which means that each supplier has constant relative risk aversion degree and risk aversion is stronger the smaller is $a$ in $(0,1)$. For regime $\mathrm{C},(6)$ yields $\hat{p}_{r}=a \hat{p}$. For the case of PR, (7) reduces to

$$
P_{r}^{\prime}(x)+\frac{n-1}{a} \frac{f(x)}{F(x)} P_{r}(x)=1
$$

and multiplying both sides of (8) by $F^{\frac{n-1}{a}}(x)$ we readily find $P_{r}(x)=\int_{0}^{x} \frac{F^{\frac{n-1}{a}}(z)}{F^{\frac{n-1}{a}}(x)} d z$. Next proposition shows that $\mathrm{C} \succ \mathrm{PR}$ when suppliers are very risk averse, regardless of the distribution.

Proposition 3 When each supplier has the utility function $u(p)=p^{a}$ and is very risk averse, that is when the parameter a is close to zero, $C$ is the best policy for $B$ for any distribution $F$ such that the profile of strategies in which each supplier bids $\hat{p}_{r}$ is a NE under $C$.

As we noticed with regard to Proposition 2, risk aversion induces lower bids for both regimes $\mathrm{C}$ and $\mathrm{PR}$, but Proposition 3 reveals that strong risk aversion has a greater effect on $\mathrm{C}$ than on $\mathrm{PR}$ and leads to $\mathrm{C} \succ \mathrm{PR}$ for any distribution. We found difficult to provide a good intuition for this result, especially because when $a$ is close to zero, both under $\mathrm{C}$ and under PR the bids are close to zero. However, it is maybe worthwhile to notice that in a sense each supplier $i$ operates

\footnotetext{
${ }^{9}$ In fact, GGD also study the full revelation policy (FR) in which B reveals $x_{1}, \ldots, x_{n}$ to each supplier, and they show that FR PR when suppliers are risk neutral. Risk aversion does not modify the suppliers' behavior under FR and thus PR $\succ$ FR by Proposition 2(ii). This justifies our focus on the comparison between C and PR.

${ }^{10}$ This is the well known intuition which applies to an auction setting in which an object is sold by using a first price auction and bidders are risk averse [see for instance Maskin and Riley (1984)].
} 
in a more risky environment in regime $\mathrm{C}$ with respect to $\mathrm{PR}$, as in regime $\mathrm{C}$ there is one more piece of information that $i$ fails to have, the own fitness parameter $x_{i}$. As a consequence, it seems conceivable that risk aversion induces more aggressive bids under $\mathrm{C}$ than under PR, as it is seen in the next example.

Example 3 Suppose that $F(x)=x^{\theta}$ with $\theta \geq 1$, so that $f$ is logconcave; then $\hat{p}_{r}=a \hat{p}=$ $\frac{(\theta n-1) a}{n(n-1) \theta^{2}}$ and $P_{r}(x)=\frac{a}{\theta(n-1)+a} x$. This shows that as $a$ is reduced from 1 to $a^{\prime} \in(0,1)$ (i) the bid under $\mathrm{C}$ is just $a^{\prime}$ times the bid when $a=1$ : the bid is reduced by the percentage $1-a^{\prime}$; (ii) under PR the bid of each type $x$ is $\frac{[\theta(n-1)+1]}{\theta(n-1)+a^{\prime}} a^{\prime}$ times the bid when $a=1$, and $\frac{[\theta(n-1)+1]}{\theta(n-1)+a^{\prime}} a^{\prime}>a^{\prime}$ : the bid is reduced by a percentage smaller than $1-a^{\prime}$; thus risk aversion favors $\mathrm{C}$ with respect to PR. Precisely, the proof of Proposition 3 establishes that $\mathrm{C} \succ \mathrm{PR}$ if and only if (16) holds, and (16) reduces to $\theta(n-1)>\left(\theta^{2} n^{2}-1\right) a$ given $F(x)=x^{\theta}$. This shows that $\mathrm{PR} \succ \mathrm{C}$ for $a$ close to 1 , but the inequality becomes less restrictive as $a$ is reduced in $(0,1)$ and there is an $a^{*} \in(0,1)$ such that $\mathrm{C} \succ \mathrm{PR}$ for $a$ between 0 and $a^{*}$, as Proposition 3 states.

\section{Appendix}

\section{Proof of the claim in footnote 5}

Given any $y>0$, Theorem 2 in CN implies that a pure strategy symmetric NE under C exists when each supplier's price is constrained to belong to $[0, y]$, provided that $f$ is logconcave. Here we prove that $\hat{\mathbf{p}}$ is a NE when there is no maximal level for prices, provided that $f$ is logconcave. Precisely, we show that (i) $\hat{\mathbf{p}}$ is a NE when each price must belong to $[0, y]$ and $y$ is larger than $\hat{p}$; (ii) $\hat{\mathbf{p}}$ is a NE also when each price can be any non negative number.

Regarding (i), suppose that $y>\hat{p}$. We know by Theorem 2 in CN that a pure strategy symmetric NE exists and the only candidates are $\hat{\mathbf{p}}$ and the profile such that each supplier bids $y$. The expected profit of supplier $i$ when each other supplier bids $y$ is $p_{i} \int_{0}^{1} F^{n-1}\left(x_{i}+y-p_{i}\right) f\left(x_{i}\right) d x_{i}$, and the derivative of this function with respect to $p_{i}$ at $p_{i}=y$ is $\int_{0}^{1} F^{n-1}\left(x_{i}\right) f\left(x_{i}\right) d x_{i}-y \int_{0}^{1}(n-$ 1) $F^{n-2}\left(x_{i}\right) f^{2}\left(x_{i}\right) d x_{i}=\frac{1}{n}\left(1-\frac{y}{\hat{p}}\right)<0$ after using (1). As a consequence, the profile such that each supplier bids $y$ is not a NE but $\hat{\mathbf{p}}$ is so in view of the existence theorem.

Regarding (ii), assume that there is no upper bound on prices and that all suppliers different from $i$ bid $\hat{p}$. Then there exists no $p_{i} \neq \hat{p}$ which yields supplier $i$ a profit higher than $i$ 's profit from bidding $\hat{p}$, because if such a $p_{i}$ existed then $\hat{\mathbf{p}}$ would not be a NE when prices need to belong to $[0, y]$ and $y>\max \left\{\hat{p}, p_{i}\right\}$, contradicting (i).

\section{Proof of the claim in footnote 6}

Consider the auction sale environment (denoted by AS in the following) in which an object is sold through a first price auction with $n$ bidders and $x_{i}$ is the valuation of bidder $i$, for $i=$ 
$1, \ldots, n$. In such a setting a symmetric BNE is characterized by a bidding function $\beta:[0,1] \rightarrow \mathbb{R}$ such that each bidder with type $x$ bids $\beta(x)$. The following result links the PR setting of GGD and the AS setting.

Lemma 1 In the PR setting of GGD, there exists a symmetric BNE in which the bidding function is $P$ if and only if there exists a symmetric BNE in the AS setting in which the bidding function is $\beta$ with $\beta(x)=x-P(x)$ for any $x \in[0,1]$.

Proof. For the PR setting of GGD, there exists a symmetric BNE in which all suppliers bid according to the function $P$ if and only if

$$
\text { for any } x_{i} \in[0,1], \quad \begin{gathered}
p_{i} \operatorname{Pr}\left\{x_{i}-p_{i}>x_{j}-P\left(x_{j}\right) \text { for any } j \neq i\right\} \\
\text { is maximized with respect to } p_{i} \text { at } p_{i}=P\left(x_{i}\right)
\end{gathered}
$$

In the AS environment there exists a symmetric BNE in which all bidders bid according to the function $\beta$ if and only if

$$
\begin{gathered}
\left(x_{i}-b_{i}\right) \operatorname{Pr}\left\{b_{i}>\beta\left(x_{j}\right) \text { for any } j \neq i\right\} \\
\text { for any } x_{i} \in[0,1], \quad \text { is maximized with respect to } b_{i} \text { at } b_{i}=\beta\left(x_{i}\right)
\end{gathered}
$$

Now we prove that $P$ satisfies (9) if and only if $\beta$ satisfies (10) with $\beta(x)=x-P(x)$. For instance, suppose that $P$ satisfies (9) and let $\beta(x) \equiv x-P(x)$, so that (10) is written as

$$
\begin{gathered}
\left(x_{i}-b_{i}\right) \operatorname{Pr}\left\{b_{i}>x_{j}-P\left(x_{j}\right) \text { for any } j \neq i\right\} \\
\text { is maximized with respect to } b_{i} \text { at } b_{i}=x_{i}-P\left(x_{i}\right)
\end{gathered}
$$

and upon using $p_{i}$ instead of $x_{i}-b_{i}$ we see that (11) is equivalent to (9). Since (9) is satisfied by assumption, we infer that also (11) holds, and so (10) is satisfied with $\beta(x)=x-P(x)$. Therefore, there exists a BNE in the AS environment in which all bidders bid according to $\beta(x)=x-P(x)$.

In a very similar manner we can prove the reverse implication: suppose that $\beta$ satisfies (10) and let $P(x) \equiv x-\beta(x)$, so that (9) is written as

$$
\text { for any } x_{i} \in[0,1], \quad \begin{gathered}
p_{i} \operatorname{Pr}\left\{x_{i}-p_{i}>\beta\left(x_{j}\right) \text { for any } j \neq i\right\} \\
\text { is maximized with respect to } p_{i} \text { at } p_{i}=x_{i}-\beta\left(x_{i}\right)
\end{gathered}
$$

and upon using $b_{i}$ instead of $x_{i}-p_{i}$ we see that (12) is equivalent to (10). Since (10) is satisfied by assumption, we infer that also (12) holds, and so (9) is satisfied with $P(x)=x-\beta(x)$. Therefore, there exists a BNE in the PR setting of GGD in which all suppliers bid according to $P(x)=x-\beta(x)$.

In the AS environment, when the bidders' values are i.i.d. with c.d.f. $F$, it is known that the unique symmetric BNE is such that $\beta(x)=x-\int_{0}^{x} \frac{F^{n-1}(z)}{F^{n-1}(x)} d z$, thus it follows from Lemma 1 that the unique symmetric BNE in GGD's PR setting is such that $P(x)=\int_{0}^{x} \frac{F^{n-1}(z)}{F^{n-1}(x)} d z$. 


\section{Proof for example 2}

Since suppliers are ex ante symmetric, we prove that $\left(p_{1}, p_{2}\right)=\left(\frac{1}{4}, \frac{1}{4}\right)$ is a NE under C by showing that given $p_{2}=\frac{1}{4}$, the profit of supplier 1 is maximized at $p_{1}=\frac{1}{4}$. The profit of 1 as a function of $p_{1}$ is $\pi_{1}\left(p_{1}\right)=p_{1} \int_{0}^{1} F\left(\frac{1}{4}+x_{1}-p_{1}\right) f\left(x_{1}\right) d x_{1}$ and $\pi_{1}\left(\frac{1}{4}\right)=\frac{1}{8}$; in the following we distinguish the case of $p_{1}<\frac{1}{4}$ from the case of $p_{1}>\frac{1}{4}$.

- For $p_{1}<\frac{1}{4}$ we find that $\frac{1}{4}+x_{1}-p_{1}>1$ for $x_{1}$ close to 1 , so that $\pi_{1}\left(p_{1}\right)=p_{1} \int_{0}^{\frac{3}{4}+p_{1}} F\left(\frac{1}{4}+\right.$ $\left.x_{1}-p_{1}\right) f\left(x_{1}\right) d x_{1}+p_{1} \int_{\frac{3}{4}+p_{1}}^{1} f\left(x_{1}\right) d x_{1}$.

If $p_{1} \leq \frac{1}{20}$, then $\pi_{1}\left(p_{1}\right)=p_{1} \int_{0}^{\frac{11}{20}+p_{1}} \frac{1}{2}\left(\frac{1}{4}+x_{1}-p_{1}\right) \frac{1}{2} d x_{1}+p_{1} \int_{\frac{11}{20}+p_{1}}^{\frac{3}{4}+p_{1}}\left(3\left(\frac{1}{4}+x_{1}-p_{1}\right)-2\right) \frac{1}{2} d x_{1}+$ $p_{1} \int_{\frac{3}{4}+p_{1}}^{\frac{4}{5}} \frac{1}{2} d x_{1}+p_{1} \int_{\frac{4}{5}}^{1} 3 d x_{1}=-\frac{1}{8} p_{1}^{3}-\frac{7}{16} p_{1}^{2}+\frac{491}{640} p_{1}$ and $\pi_{1}^{\prime}\left(p_{1}\right)=-\frac{3}{8} p_{1}^{2}-\frac{7}{8} p_{1}+\frac{491}{640}>0$ for any $p_{1} \in\left[0, \frac{1}{20}\right]$.

If $\frac{1}{20}<p_{1}<\frac{1}{4}$, then $\pi_{1}\left(p_{1}\right)=p_{1} \int_{0}^{\frac{11}{20}+p_{1}} \frac{1}{2}\left(\frac{1}{4}+x_{1}-p_{1}\right) \frac{1}{2} d x_{1}+p_{1} \int_{\frac{11}{20}+p_{1}}^{\frac{4}{5}}\left(3\left(\frac{1}{4}+x_{1}-p_{1}\right)-\right.$ 2) $\frac{1}{2} d x_{1}+p_{1} \int_{\frac{4}{5}}^{\frac{3}{4}+p_{1}}\left(3\left(\frac{1}{4}+x_{1}-p_{1}\right)-2\right) 3 d x_{1}+p_{1} \int_{\frac{3}{4}+p_{1}}^{1} 3 d x_{1}=-\frac{31}{8} p_{1}^{3}-\frac{1}{16} p_{1}^{2}+\frac{97}{128} p_{1}$ and $\pi_{1}^{\prime}\left(p_{1}\right)=-\frac{93}{8} p_{1}^{2}-\frac{1}{8} p_{1}+\frac{97}{128}>0$ for any $p_{1} \in\left(\frac{1}{20}, \frac{1}{4}\right)$.

- For $p_{1}>\frac{1}{4}$ we find that $\frac{1}{4}+x_{1}-p_{1}<0$ for $x_{1}$ close to zero, so that $\pi_{1}\left(p_{1}\right)=p_{1} \int_{p_{1}-\frac{1}{4}}^{1} F\left(\frac{1}{4}+\right.$ $\left.x_{1}-p_{1}\right) f\left(x_{1}\right) d x_{1}$.

If $\frac{1}{4}<p_{1} \leq \frac{9}{20}$, then $\pi_{1}\left(p_{1}\right)=p_{1} \int_{p_{1}-\frac{1}{4}}^{\frac{4}{5}} \frac{1}{2}\left(\frac{1}{4}+x_{1}-p_{1}\right) \frac{1}{2} d x_{1}+p_{1} \int_{\frac{4}{5}}^{\frac{11}{20}+p_{1}} \frac{1}{2}\left(\frac{1}{4}+x_{1}-p_{1}\right) 3 d x_{1}+$ $p_{1} \int_{\frac{11}{20}+p_{1}}^{1}\left(3\left(\frac{1}{4}+x_{1}-p_{1}\right)-2\right) 3 d x_{1}=\frac{159}{128} p_{1}-\frac{63}{16} p_{1}^{2}+\frac{31}{8} p_{1}^{3}$ and $\pi_{1}^{\prime}\left(p_{1}\right)=\frac{159}{128}-\frac{63}{8} p_{1}+\frac{93}{8} p_{1}^{2}<0$ for any $p_{1} \in\left(\frac{1}{4}, \frac{53}{124}\right)$ while $\pi_{1}^{\prime}\left(p_{1}\right)>0$ for any $p_{1} \in\left(\frac{53}{124}, \frac{9}{20}\right]$.

If $\frac{9}{20}<p_{1} \leq \frac{21}{20}$, then $\pi_{1}\left(p_{1}\right)=p_{1} \int_{p_{1}-\frac{1}{4}}^{\frac{4}{5}} \frac{1}{2}\left(\frac{1}{4}+x_{1}-p_{1}\right) \frac{1}{2} d x_{1}+p_{1} \int_{\frac{4}{5}}^{1} \frac{1}{2}\left(\frac{1}{4}+x_{1}-p_{1}\right) 3 d x_{1}=$ $\frac{309}{640} p_{1}-\frac{9}{16} p_{1}^{2}+\frac{1}{8} p_{1}^{3}$ and $\pi_{1}^{\prime}\left(p_{1}\right)=\frac{309}{640}-\frac{9}{8} p_{1}+\frac{3}{8} p_{1}^{2}>0$ for any $p_{1} \in\left(\frac{9}{20}, \frac{3}{2}-\frac{1}{20} \sqrt{385}\right)$, $\pi_{1}^{\prime}\left(p_{1}\right)<0$ for any $p_{1} \in\left(\frac{3}{2}-\frac{1}{20} \sqrt{385}, \frac{21}{20}\right)$. We find that $\pi_{1}\left(\frac{3}{2}-\frac{1}{20} \sqrt{385}\right) \simeq 0.11654$, which is smaller than $\pi_{1}\left(\frac{1}{4}\right)=\frac{1}{8}$.

If $\frac{21}{20}<p_{1} \leq \frac{5}{4}$, then $\pi_{1}\left(p_{1}\right)=p_{1} \int_{p_{1}-\frac{1}{4}}^{1} \frac{1}{2}\left(\frac{1}{4}+x_{1}-p_{1}\right) 3 d x_{1}=\frac{75}{64} p_{1}-\frac{15}{8} p_{1}^{2}+\frac{3}{4} p_{1}^{3}$ and $\pi_{1}^{\prime}\left(p_{1}\right)=\frac{75}{64}-\frac{15}{4} p_{1}+\frac{9}{4} p_{1}^{2}<0$ for any $p_{1} \in\left(\frac{21}{20}, \frac{5}{4}\right)$.

\section{Proof of Proposition 1}

Given (5), we have $\int_{0}^{1} g^{2}(x) d x=\int_{0}^{1} f^{2}(1-x) d x$ and the substitution $z=1-x$ yields $\int_{0}^{1} f^{2}(1-$ $x) d x=\int_{1}^{0} f^{2}(z)(-1) d z ;$ thus $\int_{0}^{1} g^{2}(x) d x=\int_{0}^{1} f^{2}(x) d x$.

In order to show that $\int_{0}^{1} F(x)[1-F(x)] d x=\int_{0}^{1} G(x)[1-G(x)] d x$, we define $\Phi(x)=F(x)[1-$ $F(x)]$ and $\Gamma(x)=G(x)[1-G(x)]$. We prove below that $\Phi(x)=\Gamma(1-x)$ for any $x \in[0,1]$, which implies $\int_{0}^{1} \Phi(x) d x=\int_{0}^{1} \Gamma(1-x) d x=\int_{0}^{1} \Gamma(x) d x$ (the latter equality is proved by using 
the substitution $z=1-x)$; thus our goal is achieved. We notice that the derivative of $\Phi$ is $f(x)[1-2 F(x)]$, while the derivative of $\Gamma(1-x)$ is $g(1-x)[2 G(1-x)-1]$, that is $f(x)[2 G(1-x)-1]$. These derivatives are equal as long as $1=F(x)+G(1-x)$, and this equality holds since $G(1-x)=\int_{0}^{1-x} g(z) d z=\int_{0}^{1-x} f(1-z) d z=\int_{x}^{1} f(t) d t$ after using the substitution $t=1-z$.

\section{Proof of Proposition 2}

(i) This proof mimics the arguments in GGD, already sketched in Section 2. We consider symmetric pure strategy $\mathrm{NE}$, in which all suppliers make the same bid $\hat{p}_{r}$ and examine the point of view of supplier $i$, who supposes that $p_{j}=\hat{p}_{r}$ for any $j \neq i$. If $i$ chooses $p_{i}$, then she wins with probability $q\left(p_{i}\right) \equiv \int_{0}^{1} F^{n-1}\left(x_{i}+\hat{p}_{r}-p_{i}\right) f\left(x_{i}\right) d x_{i}$ and she is interested in maximizing $u\left(p_{i}\right) q\left(p_{i}\right)$. The FOC with respect to $p_{i}$ is $u^{\prime}\left(p_{i}\right) \int_{0}^{1} F^{n-1}\left(x_{i}+\hat{p}_{r}-p_{i}\right) f\left(x_{i}\right) d x_{i}=u\left(p_{i}\right) \int_{0}^{1}(n-$ 1) $F^{n-2}\left(x_{i}+\hat{p}_{r}-p_{i}\right) f\left(x_{i}+\hat{p}_{r}-p_{i}\right) f\left(x_{i}\right) d x_{i}$, and since it needs to be satisfied at $p_{i}=\hat{p}_{r}$ we obtain $\frac{1}{n} u^{\prime}\left(\hat{p}_{r}\right)=u\left(\hat{p}_{r}\right) \int_{0}^{1}(n-1) F^{n-2}\left(x_{i}\right) f^{2}\left(x_{i}\right) d x_{i}$. This equality is equivalent to (6) in view of (1).

Since $u$ is concave, it is possible to prove that $\frac{u(p)}{u^{\prime}(p)}$ is strictly increasing and that $\frac{u(p)}{u^{\prime}(p)}>p$ for any $p>0$. Thus we find that $\hat{p}_{r}<\hat{p}$.

In order to establish existence when $f$ is logconcave it is useful to recall the arguments of $\mathrm{CN}$. $\mathrm{CN}$ show that (i) $f$ logconcave implies that $v\left(p_{i}\right) \equiv \frac{1}{q\left(p_{i}\right)}$ is convex in $p_{i}$; (ii) if $v$ is convex, then the payoff of supplier $i$ under risk neutrality, $p_{i} q\left(p_{i}\right)$, is quasiconcave in $p_{i}$. Given the constraint that $p_{i}$ belongs to the compact set $[0, y]$, we find that (i) and (ii) allow to apply a standard existence theorem [theorem 1.2 in Fudenberg and Tirole (1991)]. Under risk aversion, (i) still holds and thus we now prove that if $v$ is convex, then the payoff of supplier $i$ under risk aversion, $u\left(p_{i}\right) q\left(p_{i}\right)$, is quasiconcave; this is done with the following minor extension of the proof of Proposition 3 in CN. In view of a contradiction, suppose that $u\left(p_{i}\right) q\left(p_{i}\right)$ is not quasiconcave. Then there exists two prices $p_{i}^{0}$ and $p_{i}^{1}$, and $\lambda \in(0,1)$ such that $p_{i}^{\lambda} \equiv(1-\lambda) p_{i}^{0}+\lambda p_{i}^{1}$ and

$$
u\left(p_{i}^{0}\right) q\left(p_{i}^{0}\right)>u\left(p_{i}^{\lambda}\right) q\left(p_{i}^{\lambda}\right), \quad u\left(p_{i}^{1}\right) q\left(p_{i}^{1}\right)>u\left(p_{i}^{\lambda}\right) q\left(p_{i}^{\lambda}\right)
$$

Hence $q\left(p_{i}^{0}\right)>0, q\left(p_{i}^{1}\right)>0$ and we can divide the inequalities in (13) by $q\left(p_{i}^{0}\right) q\left(p_{i}^{\lambda}\right)$ and by $q\left(p_{i}^{1}\right) q\left(p_{i}^{\lambda}\right)$, respectively, to obtain

$$
\frac{u\left(p_{i}^{0}\right)}{q\left(p_{i}^{\lambda}\right)}>\frac{u\left(p_{i}^{\lambda}\right)}{q\left(p_{i}^{0}\right)}, \quad \frac{u\left(p_{i}^{1}\right)}{q\left(p_{i}^{\lambda}\right)}>\frac{u\left(p_{i}^{\lambda}\right)}{q\left(p_{i}^{1}\right)}
$$

Now we multiply the inequalities in (14) by $1-\lambda$ and $\lambda$, respectively, and add the results to get (the first inequality below follows from strict concavity of $u$ )

$$
\frac{u\left(p_{i}^{\lambda}\right)}{q\left(p_{i}^{\lambda}\right)}>\frac{(1-\lambda) u\left(p_{i}^{0}\right)+\lambda u\left(p_{i}^{1}\right)}{q\left(p_{i}^{\lambda}\right)}>u\left(p_{i}^{\lambda}\right)\left[\frac{1-\lambda}{q\left(p_{i}^{0}\right)}+\frac{\lambda}{q\left(p_{i}^{1}\right)}\right]
$$


But the inequality $\frac{1}{q\left(p_{i}^{\lambda}\right)}>\frac{1-\lambda}{q\left(p_{i}^{0}\right)}+\frac{\lambda}{q\left(p_{i}^{1}\right)}$ implied by (15) is impossible, as it violates the assumption that $v$ is convex. This implies existence when each price needs to belong to $[0, y]$, and then we can argue like in the Proof of the claim in footnote 5 above in order to establish that a symmetric pure strategy NE exists when there is no upper bound on prices.

(ii) Lemma 1 introduced above in Proof of the claim in footnote 6 holds also in the case that suppliers are risk averse: it suffices to replace $p_{i}$ and $\left(x_{i}-b_{i}\right)$ in (9) and (10) with $u\left(p_{i}\right)$ and $u\left(x_{i}-b_{i}\right)$, respectively, and the proof still applies. For the AS setting we know from Maskin and Riley (1984) that a unique symmetric BNE exists, and the equilibrium bidding function $\beta_{r}$ satisfies the differential equation $-u^{\prime}\left[x-\beta_{r}(x)\right] \beta_{r}^{\prime}(x)+(n-1) \frac{f(x)}{F(x)} u\left[x-\beta_{r}(x)\right]=0$. By replacing $\beta_{r}(x)$ with $x-P_{r}(x)$ we obtain (7).

\section{Proof of Proposition 3}

It turns out that $x_{i}-P_{r}\left(x_{i}\right)>x_{j}-P_{r}\left(x_{j}\right)$ if and only if $x_{i}>x_{j}$, hence the highest fitness supplier wins in both regimes $\mathrm{C}$ and $\mathrm{PR}$. Then we compare B's expected payments: he pays $a \hat{p}$ in $\mathrm{C}$ and $\int_{0}^{1} P_{r}(x) d F^{n}(x)=\int_{0}^{1} \int_{0}^{x} F^{\frac{n-1}{a}}(z) d z n F^{n-1-\frac{n-1}{a}}(x) f(x) d x$ in PR. The latter expression is equal to $\frac{a n}{a n-n+1} \int_{0}^{1}\left[F^{\frac{n-1}{a}}(x)-F^{n}(x)\right] d x{ }^{11}$ thus $\mathrm{C} \succ \mathrm{PR}$ if and only if

$$
\frac{n}{a n-n+1} \int_{0}^{1}\left[F^{\frac{n-1}{a}}(x)-F^{n}(x)\right] d x>\frac{1}{n \int_{0}^{1}(n-1) F^{n-2}(x) f^{2}(x) d x}
$$

Since $\lim _{a \downarrow 0} \frac{n}{a n-n+1} \int_{0}^{1}\left[F^{\frac{n-1}{a}}(x)-F^{n}(x)\right] d x=\frac{n}{n-1} \int_{0}^{1} F^{n}(x) d x$, we now prove that

$$
n^{2} \int_{0}^{1} F^{n}(x) d x \int_{0}^{1} F^{n-2}(x) f^{2}(x) d x>1
$$

which implies $\mathrm{C} \succ \mathrm{PR}$ when $a$ is close to zero. After noticing that $\int_{0}^{1} F^{\frac{n}{2}}(x)\left[F^{\frac{n-2}{2}}(x) f(x)\right] d x=$ $\int_{0}^{1} F^{n-1}(x) f(x) d x=\frac{1}{n}$, we can use the Cauchy-Schwartz inequality ${ }^{12}$ to obtain

$$
\int_{0}^{1} F^{n}(x) d x \int_{0}^{1} F^{n-2}(x) f^{2}(x) d x \geq\left(\int_{0}^{1} F^{\frac{n}{2}}(x)\left[F^{\frac{n-2}{2}}(x) f(x)\right] d x\right)^{2}=\frac{1}{n^{2}}
$$

We prove (17) by showing that the inequality in (18) is strict. If that inequality is an equality, then there exists $(\lambda, \mu) \neq(0,0)$ such that $\lambda F^{\frac{n-2}{2}}(x) f(x)=\mu F^{\frac{n}{2}}(x) d x$ for almost any $x \in(0,1)$; thus $\frac{f(x)}{F(x)}=\frac{\mu}{\lambda}$, or $F(x)=k e^{\frac{\mu}{\lambda} x}$ for some $k \in \mathbb{R}$, a contradiction since $F$ is a c.d.f.

\footnotetext{
${ }^{11}$ This is correct as long as $a \neq \frac{n-1}{n}$, and when $a$ is close to zero we can neglect the case of $a=\frac{n-1}{n}$.

${ }^{12}$ Section 6.2 in Royden (1968) introduces the so-called Hölder inequality, of which the Cauchy-Schwarz inequality is the particular case that is obtained by setting $p=q=2$ in the notation of Royden (1968).
} 


\section{References}

[1] An, M.Y., Logconcavity versus Logconvexity: A Complete Characterization, Journal of Economic Theory 80, 350-369.

[2] Anderson, S.P., A. de Palma and J.-F. Thisse, 1992, Discrete Choice Theory of Product Differentiation (The MIT Press, Cambridge, Massachusetts).

[3] Caplin, A. and B. Nalebuff, 1991, Aggregation and Imperfect Competition: On the Existence of Equilibrium, Econometrica 59, 25-59.

[4] Fudenberg, D, and J. Tirole, 1991, Game theory (The MIT Press, Cambridge, Massachusetts).

[5] Gal-Or, E., M. Gal-Or and A. Dukes, 2007, Optimal Information Revelation in Procurement Schemes, RAND Journal of Economics 38, 400-418.

[6] Maskin, E. and J. Riley, 1984, Optimal Auctions with Risk Averse Buyers, Econometrica $52,1473-1518$.

[7] Perloff, J.M. and S.C. Salop, 1985, Equilibrium with Product Differentiation, Review of Economic Studies 52, 107-120.

[8] Royden, H.L., 1968, Real Analysis (MacMillan Publishing, New York). 